\title{
Phase diagram and phase transitions in ferroelectric tris-sarcosine calcium chloride and its brominated isomorphs
}

\author{
S. P. P. Jones, ${ }^{1,{ }^{*}}$ D. M. Evans, ${ }^{1, \dagger}$ M. A. Carpenter, ${ }^{2}$ S. A. T. Redfern, ${ }^{2}$ J. F. Scott, ${ }^{1}$ U. Straube, ${ }^{3}$ and V. H. Schmidt ${ }^{4}$ \\ ${ }^{1}$ Department of Physics, Cavendish Laboratory, Cambridge University, Cambridge CB3 OHE, United Kingdom \\ ${ }^{2}$ Department of Earth Sciences, Cambridge University, Cambridge CB2 3EQ, United Kingdom \\ ${ }^{3}$ Department of Physics, University Halle, Halle, Saale D-06108, Germany \\ ${ }^{4}$ Department of Physics, Montana State University, Bozeman, Montana 59717, USA
}

(Received 24 August 2010; revised manuscript received 19 October 2010; published 4 March 2011)

\begin{abstract}
Tris-sarcosine calcium chloride [(TSCC), $\left(\mathrm{CH}_{3} \mathrm{NHCH}_{2} \mathrm{COOH}\right)_{3} \mathrm{CaCl}_{2}$ ] is a uniaxial ferroelectric (FE) with a displacive second-order phase transition near $T_{c}=130 \mathrm{~K}$. A continuous range of solid solutions can be made by substituting $\mathrm{Br}$ for $\mathrm{Cl}$, which lowers $T_{c}$ to $0 \mathrm{~K}$ at $\sim 72 \% \mathrm{Br}$. Such a quantum critical point differs from that in pseudocubic FEs, such as $\mathrm{O}-18 \mathrm{SrTiO}_{3}$ or doped $\mathrm{KTaO}_{3}$. For many years, this system was thought to have only two phases, paraelectric and FE, at ambient pressure. However, we find from dielectric and resonant ultrasound spectroscopy that there are four phase transitions in TSCC and in TSCC: $\mathrm{Br}$ (for $0<\mathrm{Br}<40 \%$ ): Order-disorder of the sarcosine methyl group at $185 \mathrm{~K}$; displacive FE transition at $130 \mathrm{~K}$ (in pure TSCC); a second FE transition [previously hypothesized to be antiferroelectric (AFE) but probably not] at $64 \mathrm{~K}$; and a new anomaly at $\sim 45 \mathrm{~K}$ which might be due to a phase transition or to Debye-like freezing of orientational disorder of some part of the sarcosine molecule. The probable sequence of structures is (upon cooling): Pnma with $Z=4\left(D_{2 h}^{16}\right)$ ambient $500 \mathrm{~K}>T>185 \mathrm{~K}$, disordered; Pnma with $Z=4\left(D_{2 h}^{16}\right) 185 \mathrm{~K}>T>130 \mathrm{~K}$ (ordered); Pn $2_{1} a$ with $Z=4\left(C_{2 v}^{9}\right) 130 \mathrm{~K}>T>64 \mathrm{~K}(\mathrm{FE}) ; P 2_{1} a\left(C_{2 h}^{5}\right)$ with $Z=4,64 \mathrm{~K}>T>45 \mathrm{~K}$ (not AFE); $T<45 \mathrm{~K}$, unknown structure. A sixth hexagonal structure at high temperatures $(>500 \mathrm{~K})$ is hypothesized to be $D_{6 h}^{3}\left(\mathrm{P}_{3} / \mathrm{mcm}\right)$ with $Z=2$, but the samples decompose first at $503 \mathrm{~K}\left(230^{\circ} \mathrm{C}\right)$.
\end{abstract}

DOI: 10.1103/PhysRevB.83.094102

PACS number(s): 61.50.Ks, 62.20.de, 64.60.-i, 77.55.fp

\section{INTRODUCTION}

Tris-sarcosine calcium chloride (TSCC) is a ferroelectric (FE) of considerable interest, with more than 100 papers devoted to its phase transition. The transition, near $130 \mathrm{~K}$, is perfectly second order, ${ }^{1}$ which is unusual among FEs. And despite its $\mathrm{N}-\mathrm{H}-\mathrm{Cl}$ hydrogen bonds, it is perfectly displacive (not order-disorder), with an underdamped soft optical phonon branch in both paraelectric (PE) and FE phases, discovered by Feldkamp and Scott in $1980 .^{2}$ The soft mode, in fact, reaches $<2 \mathrm{~cm}^{-1}$ in its underdamped state in each phase, which is second only to O-18 $\mathrm{SrTiO}_{3}$ for the softest known optical phonon. ${ }^{3}$ Prior to the soft-mode studies, it had been assumed by many authors that the transition was order-disorder, with reviewers misled ${ }^{4}$ by the unusual positive pressure dependence of Curie temperature $T_{c},{ }^{5}$ which according to Samara, ${ }^{6}$ suggests order-disorder (other exceptions, such as $\mathrm{BaMnF}_{4}$, are known $^{7}$ ). However, careful studies showed that there was no change in $T_{c}$ with deuteration, ${ }^{8}$ which is implausible for a hydrogen-bond order-disorder mechanism of FE.

The second major reason for interest in TSCC is that it forms solid solutions with $\mathrm{Br}$ substitution for $\mathrm{Cl}$, and the addition of $\mathrm{Br}$ decreases the transition temperature, reaching a quantum critical point (QCP) of $T_{c}=0$ at $72 \% \mathrm{Br}^{9}$ Since TSCC is highly uniaxial, this offers the opportunity to explore QCP FEs that, unlike $\mathrm{SrTiO}_{3}$ and $\mathrm{KTaO}_{3}$, are not pseudocubic. The pseudocubic materials exhibit dielectric constants whose reciprocals vary ${ }^{10}$ as $\left(T-T_{c}\right)^{\gamma}$, with $\gamma=2$, in contrast to the linear dependence of conventional higher-temperature FEs; but in uniaxial QCP FEs, a different exponent $\gamma$ is expected. ${ }^{11}$

We note that TSCC is a zwitter-ion crystal. The $\mathrm{COOH}$ group of molecular sarcosine has its hydrogen come off and relocate onto the $\mathrm{N}$ ion, as shown in Fig. 1, producing a symmetric $\mathrm{Cl}-\mathrm{H}-\mathrm{N}-\mathrm{H}-\mathrm{Cl}$ hydrogen-bonding structure (Fig. 2) with $\mathrm{Ca}^{+2}$ ions surrounded by six oxygen ions (three COO groups). Because the sarcosine molecules behave like stiff rods, the crystal behaves like an inorganic ionic lattice, not a soft molecular crystal. In Fig. 2, from Ashida et al., ${ }^{12}$ note the obvious pseudohexagonal symmetry; the ratio of $b$ - to $c$-lattice constants is very nearly the square root of 3 , which would be perfectly hexagonal. The $b$ axis is the polar axis, and FE is achieved by a rather rigid rotation of the sarcosine molecules out of the $a c$-mirror plane; this reaches a maximum of $\sim 3^{\circ}$ of arc at the lowest temperatures and, hence, a very small spontaneous polarization and Curie constant $(C=\sim 50 \mathrm{~K}$ compared with $10^{5} \mathrm{~K}$ in $\mathrm{ABO}_{3}$ perovskites). The rotation also stretches the $\mathrm{N}-\mathrm{H}-\mathrm{Cl}$ bonds; however, this cannot be termed an order-disorder transition because that description designates a double well whose shape is temperature independent and whose average hydrogen position $\langle x(T)\rangle$ is controlled by changing $T$, whereas, the TSCC situation is exactly opposite, with a strongly $T$-dependent length and shape for the $\mathrm{N}-\mathrm{H}-\mathrm{Cl}$ well-a classic displacive transition.

Sorge and Straube $^{13}$ performed extensive dielectric measurements at 1 bar, including dc field effects and reported calculated Landau expansion coefficients of $A_{0}=$ $(1.94 \pm 0.04) \times 10^{9} \mathrm{~V} \mathrm{~mA}^{-1} \mathrm{~s}^{-1} \mathrm{~K}^{-1}, \quad B=(4.4 \pm 0.1) \times$ $10^{15} \mathrm{Vm}^{5} \mathrm{~A}^{-3} \mathrm{~s}^{-3}$, and $C=(1.4 \pm 0.2) \times 10^{21} \mathrm{Vm}^{9} \mathrm{~A}^{-5} \mathrm{~s}^{-5}$. From adiabatic dielectric permittivity measurements at 400 $\mathrm{Hz}$ with bias fields up to $5000 \mathrm{~V} / \mathrm{cm}$, Bornarel and Schmidt ${ }^{14}$ measured $\varepsilon_{\infty}=4.2 \pm 0.2$, Curie-Weiss constant $C=38.3 \pm$ $0.3 \mathrm{~K}, T_{0}=T_{c}=129.4 \pm 0.1 \mathrm{~K}$, and calculated Landau free-energy expansion coefficients $A_{0}=(2.95 \pm 0.2) \times$ $10^{9} \mathrm{~V} \mathrm{~mA} \mathrm{~m}^{-1} \mathrm{~s}^{-1} \mathrm{~K}^{-1}$ and $B=(3.5 \pm 0.1) \times 10^{15} \mathrm{Vm}^{5} \mathrm{~A}^{-3} \mathrm{~s}^{-3}$. Brillouin scattering studies ${ }^{15,16}$ found relaxation times and 


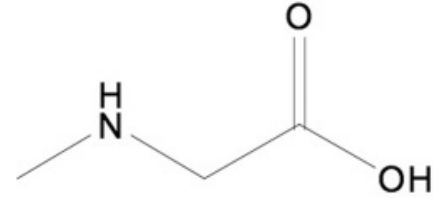

sarcosine

FIG. 1. Structure of sarcosine molecules. The carbons and hydrogens are not shown. In the crystal phase, the $\mathrm{COOH}$ ion has its $\mathrm{H}$ (zwitter ion) come off, attaching as an $\mathrm{N}-\mathrm{H}-\mathrm{Cl}$ hydrogen bond) to the other side of the $\mathrm{N}$ ion shown and leaving three polar $\mathrm{COO}$ ions around each $\mathrm{Ca}^{++}$ion.

evidence for electrostrictive coupling and for piezoelectric coupling below the 130-K transition.

Relatively recently, two new phase transitions were reported: At $185 \mathrm{~K}$, the methyl group on each sarcosine undergoes what is probably hindered (or possibly free) rotation for $T>185 \mathrm{~K}$ and fixed positions below. ${ }^{17}$ This could, of course, explain the misleading literature about order-disorder at the FE transition at $130 \mathrm{~K}$ : The FE transition at $130 \mathrm{~K}$ is displacive, but disorder sets in $55 \mathrm{~K}$ higher (but still below ambient) so that disordering does indeed occur between $\sim 125$ and $300 \mathrm{~K}$ but in two steps (transitions) rather than one. In addition, a first-order phase transition occurs at $64 \mathrm{~K} .^{18}$ Unfortunately, neither Refs. 17 nor 18 have been cited in the literature at all, so papers continue to be published without knowledge of these transitions.

It was suggested that the 64-K transition might be an antiferroelectric (AFE) phase. ${ }^{19}$ A probable AFE phase is known to exist ${ }^{20-22}$ at a few kbar pressure, with a PE-FE-AFE triple point at $176.8 \mathrm{~K}$ and $5.03 \mathrm{kbar}^{20}$ The FE-AFE phase boundary extrapolates to $0 \mathrm{~K}$ at $1.14 \mathrm{kbar},{ }^{20}$ far from the possible FE-AFE transition at $64 \mathrm{~K}$ and 1 bar. However, no extra phonon modes are observed in the high-pressure AFE phase, ${ }^{23}$ although a close inspection does show some below 64 K unmentioned by the authors of Ref. 24. No double hysteresis loops occur in the so-called AFE phase at high pressure, ${ }^{21}$ nor in the phases below $64-\mathrm{K}$, so this structure(s) might not be AFE at all. On the other hand, dielectric results ${ }^{20,21}$ indicate

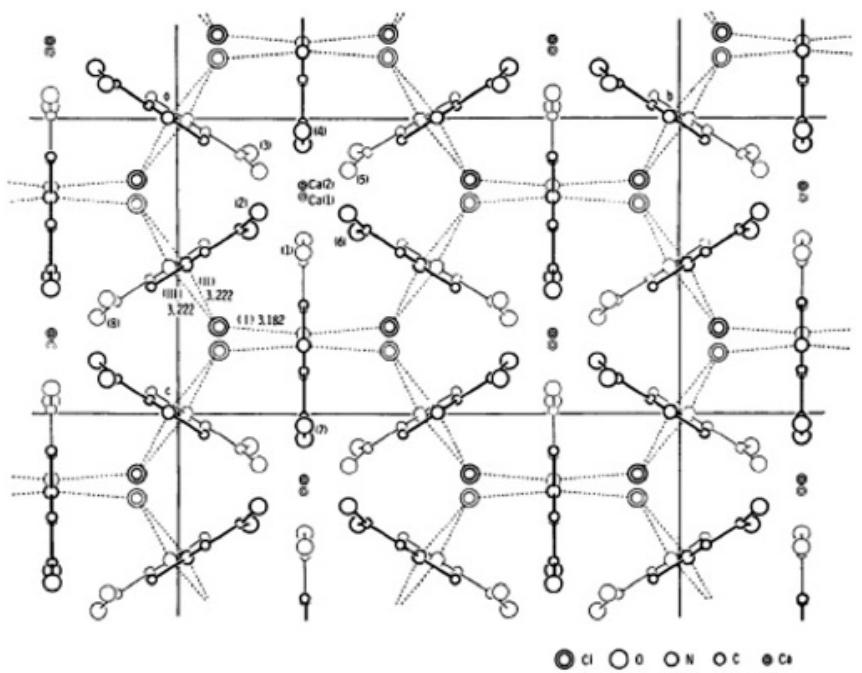

FIG. 2. Structure of TSCC, after Sawada et al. ${ }^{25}$

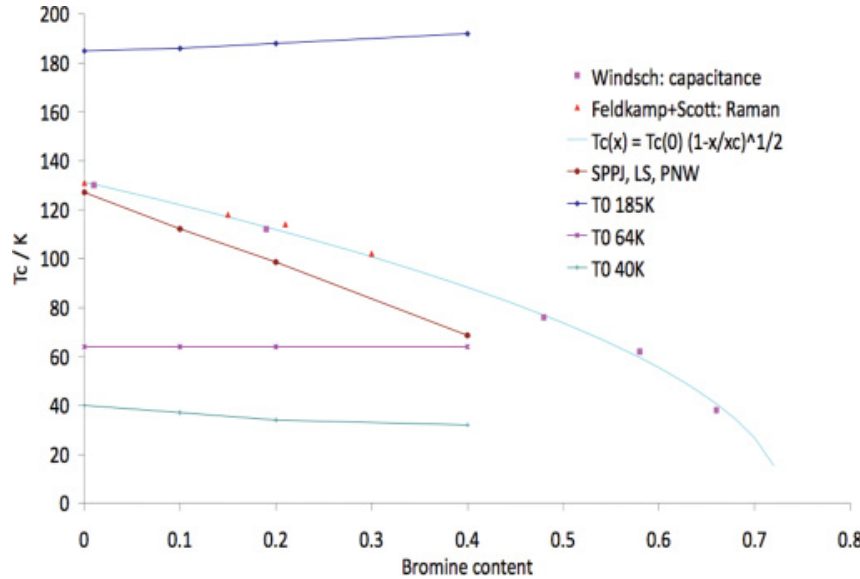

FIG. 3. (Color online) Temperature versus $\mathrm{Br}$ concentration phase diagram for TSCC and its brominated isomorphs. The newer data for brominated samples have slightly lower Curie temperatures than the older data, probably due to a difference between the starting $\mathrm{Br}$ concentration and the amount in the grown crystals. The curve is shown connecting to a value of $64 \mathrm{~K}$ at $40 \% \mathrm{Br}$, but this is probably misleading, as discussed in the text. (b) Dielectric 20\%-Br sample for cooling (peak near $103 \mathrm{~K}$ ) and heating (peak near $64 \mathrm{~K}$ ). This is not due to thermal hysteresis.

that the high-pressure FE-AFE and PE-AFE transitions are quite strongly first order, in which case, double hysteresis loops would not be expected in an AFE phase. Possible structures of the high-pressure AFE phase are orthorhombic and piezoelectric $P 2_{1} 2_{1} 2_{1}$, monoclinic and centrosymmetric $P 2_{1} / a$, and monoclinic and centrosymmetric $P 2_{1} / n$ or $P 2_{1} / b$ (described in the PE cell, or more correctly, in a new rotated cell, respectively). ${ }^{16}$ The $64-\mathrm{K}$ transition exhibits ${ }^{18}$ an entropy of transition at $1.16 \mathrm{~J} / \mathrm{mol} \mathrm{K}$, about half that exhibited at $T_{c}$, rather small for a first-order transition.

Figure 3 presents an overall phase diagram of the phases in pure TSCC and in TSCC:Br from this paper. Also, note that there is an unexplained anomaly in elastic properties at $\sim 45 \mathrm{~K}$. The saturation of $T_{c}$ with pressure at $\sim 185 \mathrm{~K}$ was noted earlier by Fujimoto et al. ${ }^{5,21}$ but not interpreted in terms of the yet-unknown methyl-disordering transition at that temperature. Finally, we have included the $D_{6 h}$ hypothesized prototype high- $T$ phase ${ }^{25}$ although samples decompose at $230{ }^{\circ} \mathrm{C}(503 \mathrm{~K})$, apparently before this hexagonal phase is reached.

\section{EXPERIMENTAL}

\section{A. Sample preparation}

The specimens used were large single crystals from earlier studies. ${ }^{13,20}$ They were oriented via external habitat and morphology (Fig. 4). They were water white and transparent; the unbrominated specimens were recrystallized by evaporation of an aqueous solution of TSCC crystals grown from $98 \%$ pure sarcosine and reagent-grade $\mathrm{CaCl}_{2} \cdot 2 \mathrm{H}_{2} \mathrm{O}$. Unlike most of the crystals grown, which were hexagonal columns composed of twins, these were lozenge-shaped single crystals. However, the brominated specimens ${ }^{13}$ were twinned hexagonal columns. 


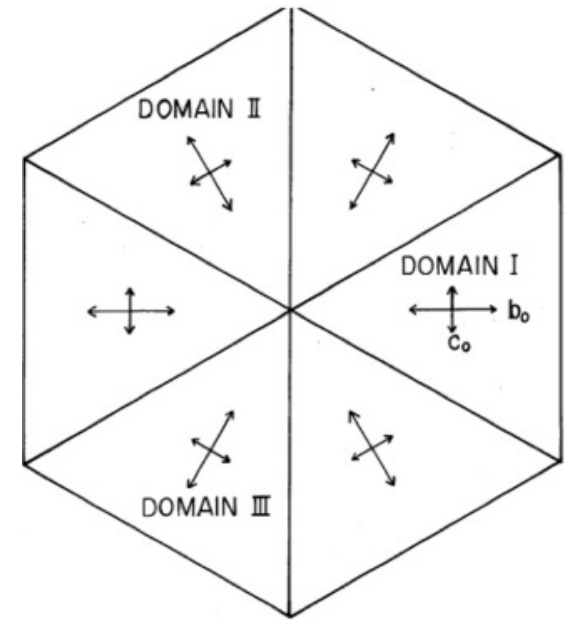

FIG. 4. Diagram of growth habitat used for orienting and cutting samples.

\section{B. Dielectric measurements}

Dielectric properties were measured from cleaved or wirecut samples, with typical dimensions of $5 \times 5 \times 0.5 \mathrm{~mm}$ and silver-paste electrodes, using an Agilent impedance analyzer at frequencies from $1 \mathrm{kHz}$ to $1 \mathrm{MHz}$ over a temperature range of $340 \mathrm{~K}$ down to $15 \mathrm{~K}$, with data taken at $1-\mathrm{K}$ intervals upon heating and cooling (typical rate: $1 \mathrm{~K} / \mathrm{min}$ ). Additional data on cooling and heating for $\mathrm{Br}$ concentrations of $0 \%, 10 \%, 15 \%$, $20 \%$, and $40 \%$ are shown in Fig. 5 for the polar $b$ axis.

The dielectric constants show a remarkable ratio of 2.00:1 for the inverse susceptibilities above and below $T_{c}$ (Fig. 6) for all samples. This is the expected textbook case for zero strain coupling but only rarely occurs in nature. This ratio confirms that the transition is second order and mean field. The exact values fitted were for $10 \%$-Br Curie constant $C=$ $20.06 \mathrm{~K}$ below $T_{c}$ and $40.12 \mathrm{~K}$ above, for $20 \%-\mathrm{Br} \mathrm{TSCC}$, $43.87 \mathrm{~K}$ above and $21.94 \mathrm{~K}$ below. $C$ is defined as $\varepsilon(T)=$ constant $+C /\left(T-T_{c}\right)$, where, for a second-order transition, the Curie-Weiss temperature (or Curie temperature) $T_{c}$ is the

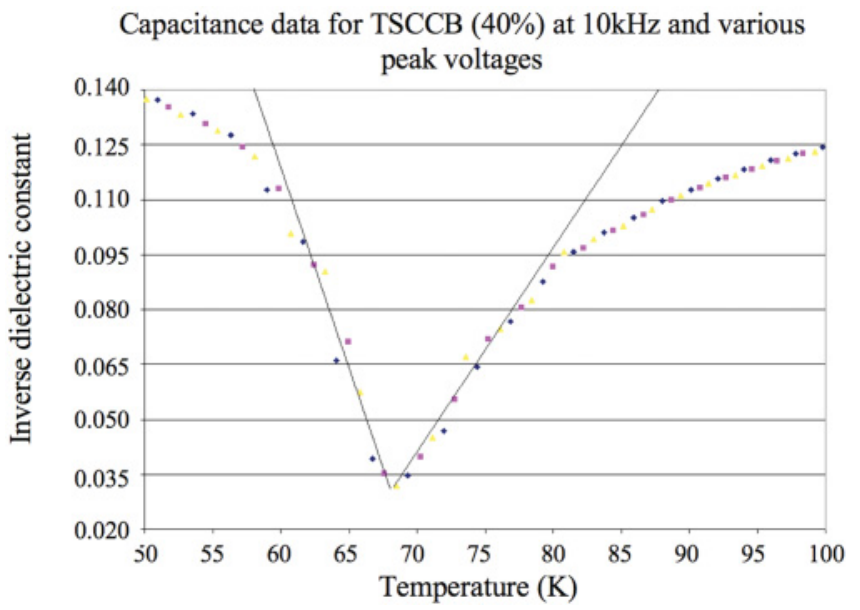

FIG. 5. (Color online) Inverse dielectric constants $1 / \varepsilon_{b}$ versus temperature, showing a ratio of 2.00:1 above and below $T_{c}$ for the $40 \%$ brominated sample. The numerical results for $10 \%$ and $20 \%$ $\mathrm{Br}$ are given in the text. Unbrominated results are given in earlier literature.
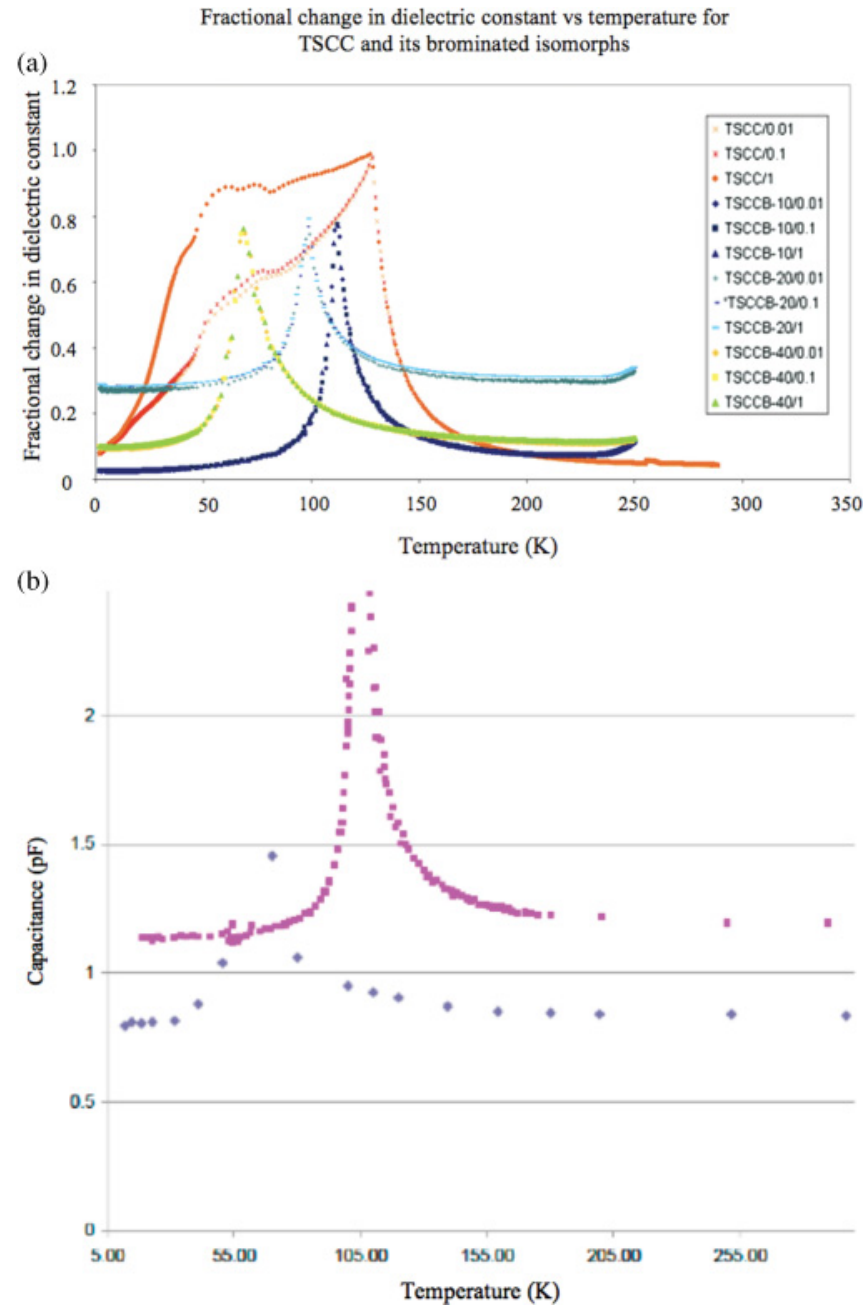

FIG. 6. (Color online) Real part of dielectric constant at $10 \mathrm{kHz}$ for different $\mathrm{Br}$ concentrations; curves at $0.01,0.1$, and $1.0 \mathrm{~V}$ applied ac test voltages. All data were in thermal equilibrium and steady state except for the pure TSCC sample, which was intentionally heated very quickly; in this case, especially at $1.0 \mathrm{~V}$, the pyroelectric effect revealed low-temperature phase transition(s) near $64 \mathrm{~K}$.

actual transition temperature. These values agree rather well with those from earlier publications (Schmidt obtained $38.3 \mathrm{~K}$ for pure TSCC compared with our $40.1 \mathrm{~K}$ for $10 \% \mathrm{Br}$ and $43.9 \mathrm{~K}$ for $20 \% \mathrm{Br}$ ). The background values of the dielectric constant of brominated TSCC were $\sim 6-8$. This is somewhat higher than the values of 4.2 for pure TSCC reported by Schmidt, which is reasonable.

One unusual aspect of the transition at $64 \mathrm{~K}$ is that it sometimes exhibits a dielectric anomaly and sometimes does not. Figure 6(b) shows this effect. A small dielectric anomaly at $64 \mathrm{~K}$ was first reported by Haga et al. ${ }^{19}$ However, we find that the dielectric anomaly can be comparable to that at $T_{c}$. What occurs is that, on cooling, the dielectric anomaly is at $T_{c}$ [105 K in Fig. 6(b)], but upon heating, it is at $64 \mathrm{~K}$. This cannot be thermal hysteresis because the sequence is backward (lower temperature upon heating, in contrast to hysteretic overshoot). It appears to be a kind of reentrant transition in which the phase between $64 \mathrm{~K}$ and $T_{c}$ is most readily obtained upon heating and can display a dielectric peak under those conditions. 


\section{Pyroelectric effects}

One of the main puzzles for us to explain about TSCC is why Haga et al. discovered the phase transition at $64 \mathrm{~K}$ via dielectric studies, whereas, equally precise measurements by other groups did not observe any anomaly near this temperature. For that reason, we examined the dielectric response, as shown in Fig. 3, for all four sample groups $(0 \%, 10 \%, 20 \%$, and $40 \%$ bromination) under various conditions. As shown, we first varied the applied ac test voltage from 0.01 to 0.1 to $1.0 \mathrm{~V}$ across each specimen. For all samples above $T_{c}$ in the PE phase, the dielectric constant was independent of voltage. And for all samples between $64 \mathrm{~K}$ and $T_{c}$, the dielectric constant was also independent of voltage if the temperature ramp rate was slow $(\mathrm{K} / \mathrm{min})$. However, as shown for the pure TSCC specimen, between $64 \mathrm{~K}$ and $T_{c}=130 \mathrm{~K}$, the dielectric constant depended strongly upon applied voltage if the heating rate was very fast $(\sim \mathrm{K} / \mathrm{s})$. We interpret this as a pyroelectric contribution to the measured dielectric constant. It is important to keep in mind that, when we measure dielectric constant, we usually measure charge. If the sample is kept at a fixed temperature for a long time (minutes), there is no contribution of pyroelectricity: We measure $\varepsilon(T)=d P(T) / d E$. However, if the heating/cooling rate is faster than the polarization relaxation time, a larger value can be measured, that is

$$
\varepsilon(T)=d P(T) / d E+(d / d E) \int[d P(T) / d T](d T / d t) d t .
$$

where $t$ is time and $d P / d T$ is the pyroelectric coefficient $p$, which is a function of $E$. If $d T / d t$ is slow, $(d / d E) p d T / d t \ll$ $d P / d E$, and there is no pyroelectric contribution.

This explains three things: (1) How Haga et al. may have discovered the transition at $64 \mathrm{~K}$ that normally has no dielectric anomaly by running data slightly faster than Windsch and others; (2) why our data in Fig. 3 depend strongly upon voltage and temperature ramp rate between $64 \mathrm{~K}$ and $T_{c}$; (3) the dielectric constant maximum is larger [top trace in Fig. 3(a)] for fast heating. In addition, and perhaps more importantly, it is independent proof that the phase(s) below $64 \mathrm{~K}$ is (are) not FE. Note, in the top trace, that there is a small peak at $64 \mathrm{~K}$ and also one at $74 \mathrm{~K}$; the $64-\mathrm{K}$ transition is slightly first order, and this may be due to thermal hysteresis (other anomalies are also found at $74 \mathrm{~K}$ ), alternatively, it could involve subsidiary dielectric anomalies due to domains. ${ }^{26}$

This figure shows, rather remarkably, the fact that, for the brominated specimens (not pure TSCC), the dielectric peak at $64 \mathrm{~K}$ disappears for slow $(\mathrm{K} / \mathrm{min})$ cooling runs [as in Fig. 3(b)]. However, more surprisingly, the main dielectric peak at $T_{c}$ disappears for fast $(\mathrm{K} / \mathrm{s})$ heating runs. This is related to but different from the anomaly discussed for Fig. 3. In this case, the key step seems to be whether or not the samples are cooled below $32 \mathrm{~K}$. If so, then on heating, the dielectric anomaly is only at $64 \mathrm{~K}$ and not at $T_{c}$. As in Fig. 3 , the dielectric peak at $64 \mathrm{~K}$ is presumably pyroelectric. However, the absence of the peak at $T_{c}$ requires further explanation; note that pyroelectricity requires piezoelectricity. An a priori possibility is that the polar axis has rotated. Although TSCC is a uniaxial FE (and not pseudocubic, like perovskites), it is pseudohexagonal (Fig. 2) and ferroelastic. Therefore, it may

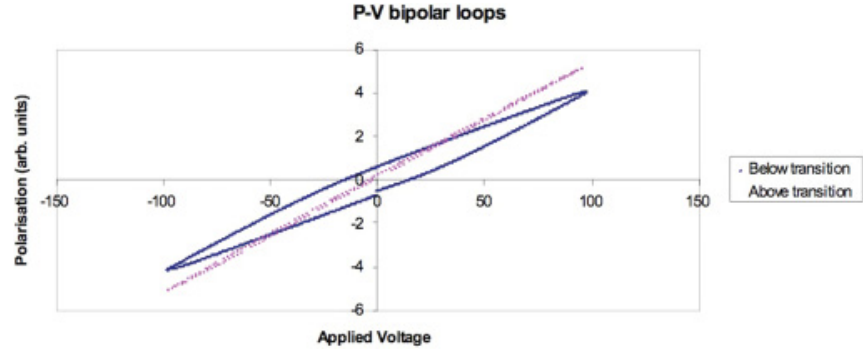

FIG. 7. (Color online) Unsaturated polarization $P(E)$ at $330 \mathrm{kV} / \mathrm{m}$ along the polar axis of pure TSCC. The lossless curve above $T_{c}=130 \mathrm{~K}$ returns below $64 \mathrm{~K}$.

be plausible that strain caused by the lower-temperature phase transition rotates the polarization from $b$ to $c$ and that the phase between $64 \mathrm{~K}$ and $T_{c}$ is $\mathrm{FE}$, but with $P$ along a different axis. This would be analogous to the phases of $\mathrm{BaTiO}_{3}$. In addition, and perhaps more importantly, it is independent proof that the phase(s) below $64 \mathrm{~K}$ is (are) not FE-at least not along the $b$ axis. However, it is quite possible that the 64-130-K phase is FE with $P$ rotated along a different axis (polar $b$ and nonpolar $c$ are very nearly equivalent in this pseudohexagonal ferroelastic). (We return to this point below in the discussion of Fig. 7.) Finally, it is important to note that the axis switching hypothesized for Fig. 7 occurs only in the multidomain hexagonal columnar specimens from Halle and not in the single-domain sample from Montana. Apparently, the piezoelectric stress is sufficient to pole the former. For switching in twinned samples, see Ref. 27.

\section{Polarization measurements}

A Radiant Technology tester was employed for polarization measurements below $130 \mathrm{~K}$, but saturated loops were not observed up to $100 \mathrm{~V}$ across $0.3 \mathrm{~mm}-3.3 \mathrm{kV} / \mathrm{cm}$ (Fig. 8), only an increase in loss below $T_{c}$. Below $64 \mathrm{~K}$, the loss disappears. Earlier work has shown that no double loops are observed in the so-called AFE phase, but the strong first-order nature

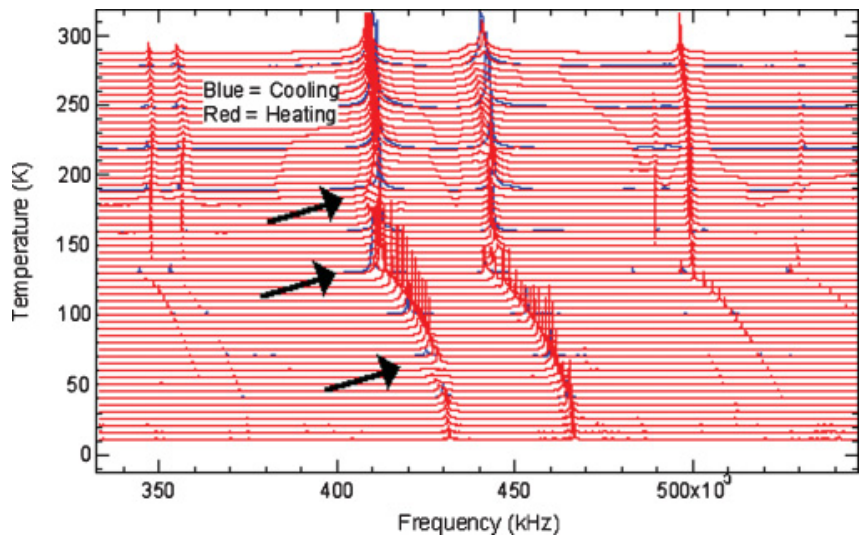

FIG. 8. (Color online) Resonant ultrasonic response versus frequency at different temperatures for TSCC. The stack of spectra reveals anomalies in elastic coefficients $C_{i j}(T)$ in the $100-\mathrm{kHz}$ regime and is very sensitive to nonpolar phase transitions. Data near temperatures at which anomalies occur in vibrational frequency or damping are indicated by arrows. 


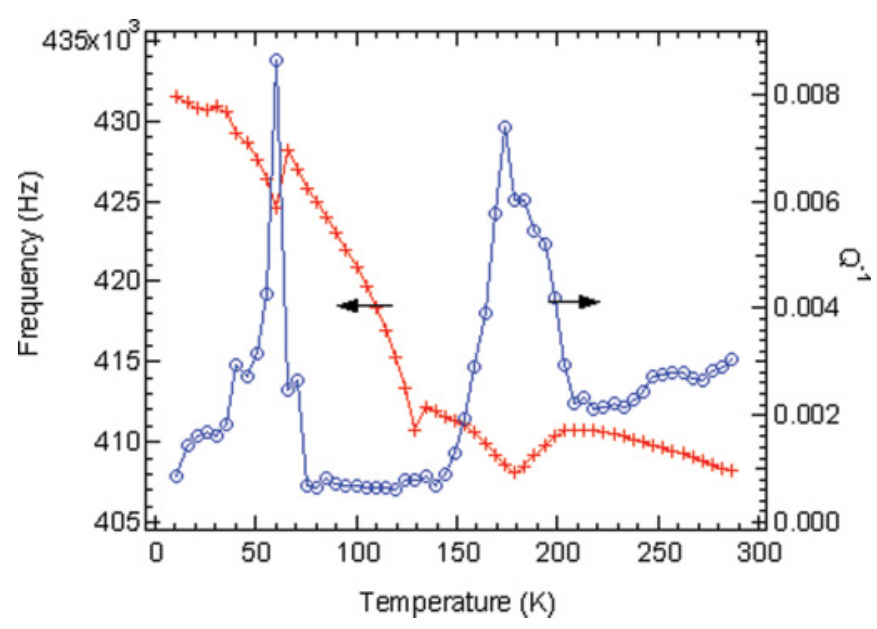

FIG. 9. (Color online) Resonant ultrasonic frequency for one mode near $420 \mathrm{kHz}$ and its loss versus temperature for TSCC.

of the FE and PE phases to that phase would likely prevent observation of such loops even if that phase is AFE.

\section{E. Resonant ultrasonic spectroscopy}

Resonant ultrasonic spectroscopy (RUS) measurements ${ }^{28}$ in the several-hundred kilohertz regime were made on free-standing cleaved $b$-face platelets, which revealed weak anomalies in elastic constants (Fig. 7) at 185, 130 (pure TSCC), 64 , and $\sim 45 \mathrm{~K}$ and very strong mechanical losses at 185 and $64 \mathrm{~K}$ (Fig. 9). Other resonance modes show strong anelastic loss also at the $130-\mathrm{K}$ transition. The phase transition at $185 \mathrm{~K}$ in pure TSCC increases in frequency with bromination, reaching $192 \mathrm{~K}$ at $40 \% \mathrm{Br}$. The dissipation peak at $\sim 45 \mathrm{~K}$ in pure TSCC also has been observed at $\sim 33 \mathrm{~K}$ in the $40 \% \mathrm{Br}$ sample.

\section{F. Dynamic mechanical analysis}

Dynamic mechanical analysis (DMA) is complementary to RUS because it works in the region of a few hertz, rather than hundreds of kilohertz. ${ }^{29}$ DMA applies a variable force on a crystal of known width $w$, length $l$, and thickness $t$; all mea- sured, in our paper, with calipers. The DMA works on the basis of a three-point bending geometry whereby a sample is placed across two knife edge supports with a force applied between them. A constant force is applied to keep the sample in contact with the knives and a dynamic force $F_{D}$ with a frequency $v$. The amplitude $(u)$ and the phase lag $(\delta)$ of the mechanical response were measured using electromagnetic inductive coupling with resolutions $\Delta u=10 \mathrm{~nm}$ and $\Delta \delta \cong 0.1^{\circ}$. Because our sample is much longer than wide, we see only the Young's $(Y)$ modulus and not the shear modulus. These are all related by

$$
Y=\frac{l^{3} F_{D} \exp (i \delta)}{4 t^{3} w u_{D}} .
$$

We examined several different frequencies for the pure TSCC specimens and show results in Fig. 10 at frequencies of 10 and $5 \mathrm{~Hz}$ because these gave the best signal-to-noise ratios. Initially, we cooled the sample at $10 \mathrm{~K} / \mathrm{min}$ but found that noise was greatly reduced if we cooled at $5 \mathrm{~K} / \mathrm{min}$. After some optimization tests, it was decided to use a static force of $600 \mathrm{mN}$ and a dynamic force of $480 \mathrm{mN}$. The primary results are as follows: For pure TSCC, the modulus shows a distinct change in slope at $T_{c}=135 \pm 5 \mathrm{~K}$, decreasing from 8 to $\sim 6 \mathrm{GPa}$ as the sample is cooled below $T_{c}$ to $\sim 100 \mathrm{~K}$. The loss tangent increases from $13 \%$ to $19 \%$ as $T$ decreases over this range, probably due to domain motion. Note that the RUS data in Fig. 9 may be sensitive to quite different elastic coefficients $C_{i j}(T)$ than the three-point bending measurements in Fig. 10, so the data near $185 \mathrm{~K}$ are complementary.

\section{G. Pressure versus temperature phase diagram}

Figure 11 illustrates the previously known $P$-versus $T$-phase diagram for pure TSCC. It can be seen, from Fig. 11 that, at several kbar of hydrostatic pressure, the FE and PE phases transform into a phase labeled AFE. There are two things worthy of note here: First, as discussed above, there is no indication that this phase is AFE; no double hysteresis loops have been reported, and no extra Raman lines are found that would signify doubling of the primitive crystallographic unit cell. Second, the phase boundary bends over with increasing pressure near

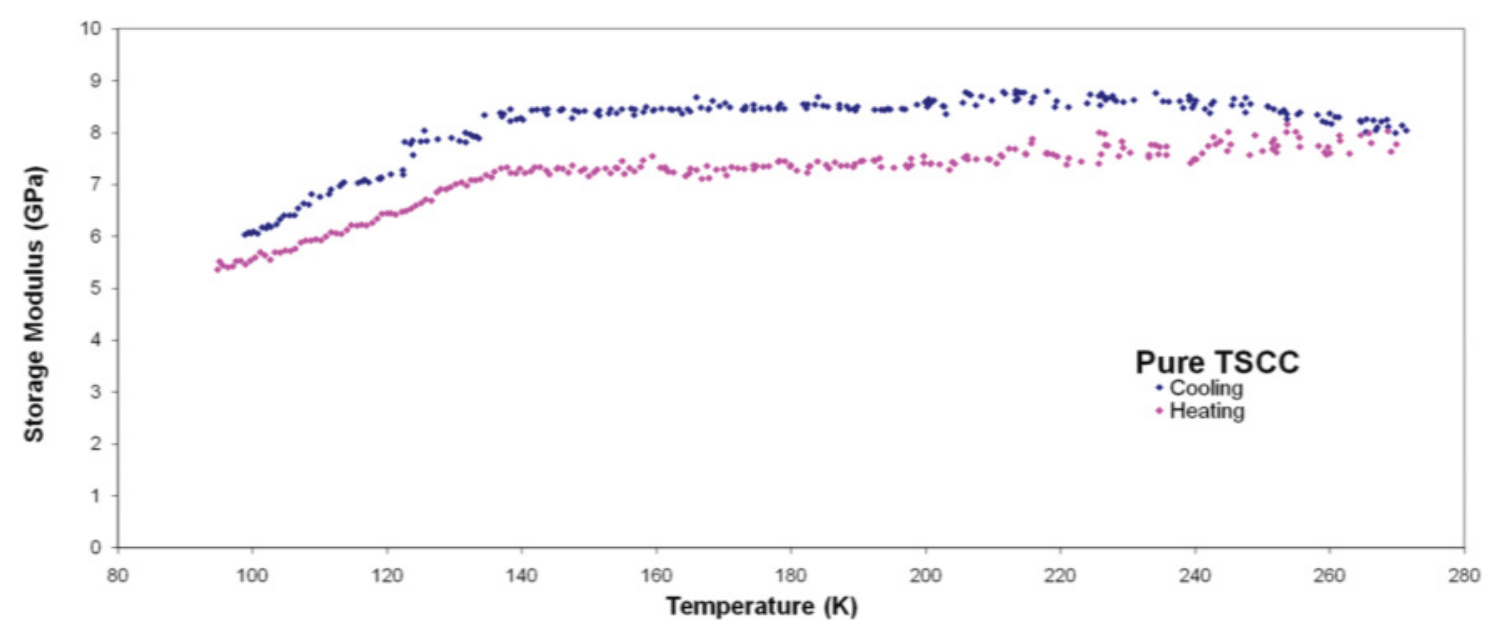

FIG. 10. (Color online) DMA results for Young's modulus. Each set of runs produced approximately 20000 data points. Excel was used to average every hundred points, and it is these averages that are plotted. 


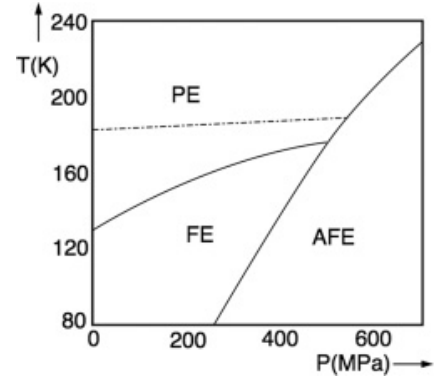

FIG. 11. Temperature versus pressure phase diagram for pure TSCC (after Refs. 18, 20, and 21), showing hypothetical interaction of the order-disorder phase near $185 \mathrm{~K}$ with the FE phase. The dashed line assumes that the $185-\mathrm{K}$ phase boundary at atmospheric pressure is pressure independent. Speculation is not made at higher pressures in the AFE phase, and the nature of the vertices is quite unknown.

$185 \mathrm{~K}$ and saturates. This temperature matches closely to that of the newly observed order-disorder phase transition, and, hence, it suggests that there may be either two closely spaced triple points or else a point where four phases come together. The curvature suggests that the order parameters for the PE/FE transition and for the $\mathrm{CH}_{3}$-rotation order-disorder transition are coupled. Rather more precise data near $185 \mathrm{~K}$ will be required to sort this out. The apparent interaction between the phase boundary at $185 \mathrm{~K}$ and the PE-FE boundary near $130 \mathrm{~K}$ seems reasonable in terms of simple mechanics: The 185-K transition involves free or hindered rotation of the methyl end group on each sarcosine. As temperature decreases below $185 \mathrm{~K}$, this probably destabilizes the position of the $\mathrm{Cl}-\mathrm{H}-\mathrm{N}-\mathrm{H}-\mathrm{Cl}$ hydrogen bonds on the adjacent sarcosine atoms such that their planar positions rotate out of the $b$ plane. At the lowest temperatures, this rotation angle reaches $\sim 3^{\circ}$ of arc, similar to that of the $\mathrm{Ti}-\mathrm{O}_{3}$ or $\mathrm{Al}-\mathrm{O}_{3}$ bonds in $\mathrm{SrTiO}_{3}$ or $\mathrm{LaAlO}_{3}$.

Experiments on deuterated TSCC and its effect on the 185-K transition will be published separately.

The other question (Fig. 11) is whether the AFE phase is the stable phase at low temperatures and zero applied pressure. Extrapolating the existing curve to temperatures below liquid nitrogen suggests that it does not intersect the transition at $T=64 \mathrm{~K}$ and $P=0$ (which has been suggested in earlier literature). As above, some further cryogenic work at liquid-He temperatures is required. In general, the presence of a phase boundary at $T=32 \mathrm{~K}$ and $P=0$ raises questions about the complexity of and even the presence of a quantum critical point at $T=0, P=0$, and $72 \% \mathrm{Br}$. The data in Fig. 3 show that, at $40 \% \mathrm{Br}$, the lowest elastic anomaly (at $\sim 45 \mathrm{~K}$ in pure TSCC) is observed at $\sim 33 \mathrm{~K}$. The previously published low-temperature data on TSCC do not go below $33 \mathrm{~K}$, although high-pressure data on $100 \%$ brominated TSCC show a FE phase and, hence, a QCP.

\section{QCPS}

The presence of unexpected anomalies at low temperatures has complicated and has delayed our study of the QCP near $72 \%$ - Br concentration. This will be discussed in a separate paper.
*Present address: Department of Physics, Oxford University, Oxford OX1 3PU, U.K.

${ }^{\dagger}$ Present address: Department of Physics, Queens University, Belfast BT7 1NN, Northern Ireland.

${ }^{1}$ Y. Makita, J. Phys. Soc. Jpn. 20, 2073 (1965).

${ }^{2}$ G. V. Kozlov, A. A. Volkov, J. F. Scott, and J. Petzelt, Phys. Rev. B 28, 255 (1983); G. E. Feldkamp and J. F. Scott, Ferroelectrics 25, 171 (1980); G. E. Feldkamp, J. F. Scott, and W. Windsch, ibid. 39, 1163 (1981); S. D. Prokhorova, G. A. Smolensky, I. G. Siny, E. G. Kuzminov, V. D. Mikvabia, and H. Arndt, ibid. 25, 629 (1980).

${ }^{3}$ M. Takesada, M. Itoh, and T. Yagi, Phys. Rev. Lett. 96, 227602 (2006).

${ }^{4}$ M. Fujimoto, S. Jerzak, and W. Windsch, Phys. Rev. B 34, 1668 (1986).

${ }^{5}$ S. Fujimoto, N. Yasuda, K. Takagi, P. S. Narayanan, and H. L. Bhat, J. Phys. D 13, L107 (1980).

${ }^{6}$ G. A. Samara, Phys. Rev. B 1, 3777 (1970).

${ }^{7}$ G. A. Samara and P. M. Richards, Phys. Rev. B 14, 5073 (1976).

${ }^{8}$ M. K. Gergs, D. Michel, H. Schlemmbach, and W. Windsch, J. Phys. D 19, 2431 (1986).

${ }^{9}$ W. Windsch, H. Braeter, U. Gutteck, B. Malige, and B. Milsch, Sol. St. Commun. 42, 839 (1982).

${ }^{10}$ U. T. Hoechli and L. A. Boatner, Phys. Rev. B 20, 266 (1979).

${ }^{11}$ Stephen Rowley, Ph.D. thesis, Cambridge University, 2011.

${ }^{12}$ T. Ashida, S. Bando, and M. Kakudo, Acta Crystallogr., Sect. B 28, 1560 (1972).

${ }^{13}$ G. Sorge and U. Straube, Phys. Status Solidi A 51, 117 (1979).
${ }^{14}$ J. Bornarel and V. H. Schmidt, J. Phys. C 14, 2017 (1981).

${ }^{15}$ T. Hikita, P. Schnackenberg, and V. H. Schmidt, Phys. Rev. B 31, 299 (1985).

${ }^{16}$ J. T. Wang and V. H. Schmidt, Phys. Rev. B 34, 416 (1986).

${ }^{17}$ H. Haga, A. Onodera, H. Yamashita, and Y. Shiosaki, Ferroelectrics 159, 55 (1994).

${ }^{18}$ K. Lee, M. Lee, K. S. Lee, and A. R. Lim, J. Phys. Chem. Solids 66, 1739 (2005).

${ }^{19}$ H. Haga, A. Onodera, H. Yamashita, and Y. Shiosaki, J. Phys. Soc. Jpn. 62, 1857 (1993).

${ }^{20}$ V. H. Schmidt, Solid State Commun. 35, 649 (1980).

${ }^{21}$ S. Fujimoto, N. Yasuda, K. Takagi, P. S. Narayanan, and H. L. Bhat, J. Phys. D 13, L217 (1980).

${ }^{22}$ Z. X. Bao, V. H. Schmidt, and F. L. Howell, Ferroelectrics 145, 241 (1993).

${ }^{23}$ R. Roth, G. Schaack, and H. D. Hochheimer, Solid State Commun. 55, 121 (1985).

${ }^{24}$ T. Chen and G. Schaack, J. Phys. C 17, 3821 (1984).

${ }^{25}$ A. Sawada, Y. Makita, and Y. Takagi, J. Phys. Soc. Jpn. 42, 1918 (1977).

${ }^{26}$ A. A. Baski, W. F. Oliver, and J. F. Scott, Phys. Rev. B 80, 214112 (2009).

${ }^{27}$ J. Bornarel, J. Lajzerowicz, and J. F. Legrand, Ferroelectrics 7, 313 (1974).

${ }^{28}$ M. A. Carpenter et al., J. Appl. Phys. 107, 013505 (2010).

${ }^{29}$ S. A. T. Redfern, C. Wang, G. Catalan, J.-W. Hong, and J. F. Scott, J. Phys.: Condens. Matter 20, 452205 (2008). 